\title{
sur la gestion et les finances publiques En Septembre 2021
}

\section{Budget DE L'ÉTAT ET DES OPÉRATEURS}

\section{$\pi$ Données générales sur les finances publiques}

Le secrétariat permanent du Haut Conseil des finances publiques publie une note méthodologique sur le PIB et la croissance potentiels

Dans le cadre du mandat que lui a confié la loi organique, le Haut Conseil des finances publiques doit donner un avis sur les estimations de produit intérieur brut potentiel (PIB potentiel) présentées par le Gouvernement. Dans un document publié le 9 septembre 2021, les deux auteurs de la note, membres du Haut Conseil des finances publiques, affirment que la notion de PIB potentiel et de croissance potentielle joue un rôle essentiel, bien que peu relayé dans le débat public, dans l'élaboration des prévisions économiques de moyen terme et dans l'évaluation de la politique budgétaire.

Dans le cadre de la programmation des finances publiques, le PIB potentiel détermine en effet les ressources financières dont pourra espérer disposer la France pour financer ses politiques publiques et sociales. L'évaluation du PIB potentiel suppose d'isoler dans le PIB observé une composante permanente et une composante transitoire. Du fait des chocs, de nature et d'amplitude diverses, qui affectent le PIB, cette évaluation n'est pas automatique et différentes méthodes peuvent être mobilisées à cet effet. L'écart de production, entre le PIB potentiel et le PIB effectif, constitue un indicateur de la capacité de rebond ou de ralentissement économique. II permet ainsi d'isoler dans le déficit effectif une part transitoire, liée à la partie purement conjonc- turelle du PIB, et d'en déduire par solde, après retrait des éléments exceptionnels, une composante "structurelle » (déficit structurel ou excédent structurel), une notion au cœur de l'architecture française et européenne des finances publiques.

\section{Procédure budgétaire}

Le Haut Conseil a adopté un avis relatif aux projets de lois de finances et de financement de la sécurité sociale pour l'année 2022

Le 22 septembre 2021, le Haut Conseil des finances publiques a publié son avis du 17 septembre 2021 relatif aux projets de lois de finances et de financement de la sécurité sociale pour l'année 2022.

Pour commencer, le Haut Conseil relève que le projet de loi de finances pour 2022 dont il a été saisi est incomplet. En effet, il n'intègre pas l'impact de mesures d'ampleur qui ont pourtant déjà été annoncées par le Gouvernement (grand plan $d^{\prime}$ investissement, revenu d'engagement notamment) et que celui-ci prévoit de faire adopter par voie d'amendement au cours du débat parlementaire.

Le Haut Conseil regrette ces conditions de saisine qui ne lui permettent pas de rendre un avis pleinement éclairé sur les prévisions de finances publiques pour 2022 à l'intention du Parlement et des citoyens, en application de son mandat. $\mathrm{Si}$, comme il est vraisemblable, le scénario macroéconomique et de finances publiques était modifié pour prendre en compte ces mesures, une nouvelle saisine du Haut Conseil par le Gouvernement serait alors nécessaire. Le Haut Conseil considère que l'hypothèse de croissance du Gouvernement pour 2021 (+ 6,0\%), révisée en hausse depuis la $1^{\text {re }}$ loi de finances rectificative de l'année du fait d'une amélioration plus 
forte qu'attendu des indicateurs conjoncturels, est prudente et que celle pour 2022 (+ 4,0\%) est plausible. La prévision d'inflation est réaliste pour 2021 et 2022. En revanche, le Haut Conseil juge les prévisions d'emploi et de masse salariale pour 2021, qui ne tiennent pas compte des toutes dernières informations disponibles, trop basses. Partant d'un niveau plus élevé en 2021, l'emploi et la masse salariale devraient également être plus élevés en 2022 que prévu par le PLF. Du fait d'un probable surcroît de recettes qui en résulterait, le déficit public pour 2021 pourrait être moins dégradé que prévu par le Gouvernement (- 8,4 points de PIB). Pour 2022, les recettes assises sur les salaires pourraient de même être sous-estimées. À l'inverse, la prévision des dépenses est raisonnable compte tenu des éléments transmis au Haut Conseil. Cependant, ces éléments étant incomplets, le Haut Conseil n'est pas à ce stade en mesure de se prononcer sur la plausibilité de la prévision de déficit pour 2022 (- 4,8 points de PIB).

Le Haut Conseil constate que le solde structurel présenté s'établirait à - 3,7 points de PIB en 2022, en appliquant I'hypothèse de PIB potentiel de la loi de programmation (LPFP) de janvier 2018. Le solde structurel serait alors dégradé de 2,9 points par rapport à celui prévu par la LPFP pour l'année 2022, soit un écart important au sens de la loi organique de 2012. Néanmoins, le Haut Conseil a estimé au printemps 2020 que les conditions économiques très particulières liées à la crise sanitaire étaient réunies pour déclencher la clause des circonstances exceptionnelles, et considère que celles-ci sont encore de nature à justifier en 2022 des écarts à la trajectoire programmée. Le Haut Conseil rappelle que la LPFP constitue une référence dépassée pour apprécier la trajectoire de finances publiques. La dernière estimation de croissance potentielle du Gouvernement, révisée pour tenir compte des conséquences de la crise sanitaire, conduit à un solde structurel plus dégradé, s'établissant à $-4,7$ points de PIB soit un écart de plus de 4 points à l'objectif de moyen terme des finances publiques que s'était donné la France dans la LPFP.

Enfin, le Haut Conseil note que le poids des dépenses publiques dans le PIB serait en 2022 près de deux points au-dessus de son niveau de 2019 en dépit du net rebond de l'activité et de l'extinction de la plupart des dépenses engagées pour répondre à la crise sanitaire. De surcroît, les mesures d'allègement d'impôts des dernières années devraient peser durablement sur les recettes. Face à un endettement public accru (114 points de PIB en 2022, soit 17 points de plus qu'en 2019), le Haut Conseil souligne que la soutenabilité à moyen terme des finances publiques appelle à la plus grande vigilance. Dans ce contexte, il importera que tout surcroît de recettes par rapport à la prévision soit consacré au désendettement.

\section{Le projet de loi de finances 2022}

Enregistré à la présidence de l'Assemblée nationale le 22 septembre 2022, le projet de loi de finances 2022 illustre une normalisation progressive des finances publiques, en dépenses et en recettes, au regard de la gestion d'urgence due à la crise sanitaire de 2020 et 2021.

La reprise économique amorcée depuis le printemps devrait permettre une croissance toujours soutenue en 2022 (+ $4 \%$ ) après un fort rebond en 2021 (+ 6 \%). Grâce à ce rebond annoncé, le déficit public devrait diminuer de l'ordre de 3,5 points de PIB, passant de - 8,4 \% en 2021 à - 4,8 \% du PIB en 2022. II est donc prévu un déficit public presque divisé par deux par rapport à 2020 .

Ainsi, la dette devrait légèrement reculer l'an prochain, avec un ratio autour de 114 points de $P I B$, après une progression très forte en 2020 à 115 points de PIB (+17,5 points par rapport à 2019) et une quasi stabilisation en 2021 (115,6 points).

Concernant les recettes, celles-ci devraient bénéficier du rebond de l'activité en 2021-2022. En 2022, les recettes de l'État devraient poursuivre leur croissance grâce à la normalisation de la situation économique. Les recettes fiscales nettes s'établiraient à $292 \mathrm{Mds} €$, avec une hausse de 13,4 Mds€ par rapport à 2021, principalement portées par l'impôt sur le revenu (+5,3 Mds€), la TVA $(+5,1 \mathrm{Mds} €)$ et l'impôt sur les sociétés $(+3,1 \mathrm{Mds} €)$. Grâce au double effet de ralentissement de la dépense et de la dynamique des recettes, le solde budgétaire pourrait sensiblement s'améliorer en 2022 selon Bercy, ce qui reste à vérifier en pratique.

\section{$\pi$ Administrations budgétaires}

Trois audits flash sur les mesures de soutien dans le secteur de la culture pendant la crise sanitaire

Le 29 septembre 2021, la Cour des comptes a publié trois audits flash analysant les mesures prises dans le cadre du soutien apporté par l'État à 3 secteurs de la culture lors de la crise de la Covid-19 : le cinéma, le patrimoine et le spectacle vivant. 
L'effort financier consenti en faveur du cinéma et de l'audiovisuel est exceptionnel : 402 M€ pour le Centre national du cinéma et de l'image animée (soit environ neuf mois de dépenses de cet organisme en 2019) et 51,6 M€ pour l'I I'Institut pour le financement du cinéma et des industries culturelles. La Cour remarque cependant que I'articulation de ces financements sectoriels additionnels avec les dispositifs généraux d'indemnisation mis en place par l'État (1,2 Mds versés aux entreprises du secteur) et le contrôle d'éventuels effets d'aubaine ont été peu développés. Constatant enfin que les dépenses participant d'une véritable logique d'investissement d'avenir s'avèrent peu élevées (entre 20 et $25 \mathrm{M} €$ ), elle recommande que le dispositif public se réoriente dorénavant vers la préparation de l'avenir : la constitution d'entreprises plus solides, le développement des infrastructures techniques de haut niveau, I'export, I'attractivité de la France.

Le montant total des soutiens budgétaires exceptionnels octroyés au patrimoine par le ministère de la culture en 2020 et 2021 est de l'ordre de $710 \mathrm{M} €$ de crédits (en autorisation d'engagement), ou de $870 \mathrm{M} €$ si l'on y ajoute la contribution exceptionnelle d'autres ministères via leurs crédits ordinaires ou le plan de relance. S'agissant des plus grands musées et monuments, leur modèle économique a été mis à mal par la désertion de la fréquentation touristique étrangère. Poursuivi, et même amplifié par le plan de relance en 2021-2023 (327,5 M€), I'accompagnement de ces opérateurs est particulièrement coûteux et pourra difficilement être pérennisé. En outre, compte tenu des incertitudes qui pèsent sur les perspectives de fréquentation touristique des lieux patrimoniaux ouverts au public, les plus grands opérateurs, jusqu'alors autofinancés à hauteur de $50 \%$, vont devoir approfondir une réflexion, déjà engagée sur les évolutions nécessaires de leur modèle économique.

À la mi-mai 2021, le ministère de la culture a mobilisé $823 \mathrm{M} €$ de crédits exceptionnels pour le spectacle vivant - en plus des dispositifs transversaux dont ont bénéficié, comme toute autre, les entreprises du secteur, et de "l'année blanche » accordée aux intermittents du spectacle via I'Unédic. Relayé au niveau local par les DRAC et, sectoriellement, par le Centre national de la musique et l'Association pour le soutien du théâtre privé, le rôle du ministère a été essentiel durant la crise. Si la mobilisation budgétaire, mise en place dans l'urgence, a été forte, les mesures prises ont été peu accompagnées de dispositifs d'évaluation ex post. II semble en outre que la multiplicité des aides a nui à leur lisibilité et n'a donc pas fa- cilité leur identification par tous les bénéficiaires potentiels. L'audit de la Cour évoque enfin l'importance des risques et des enjeux inhérents à la phase de reprise. Du fait de la persistance des effets de la crise, et malgré la probable inscription dans la durée de certaines mesures de soutien, le risque de disparition progressive d'artistes et de structures, y compris d'acteurs très installés du secteur, l'amène à souligner que le cadencement des sorties des dispositifs d'aide et leur adaptation ciblée aux acteurs les plus exposés conditionne, pour une part importante, le succès de la reprise.

\section{$\pi$ Budget des pouvoirs publics}

\section{Les dépenses du cabinet du ministre des outre-mer}

La Cour des comptes a contrôlé les dépenses de cabinet des ministres des outre-mer entre 2017 et 2020. Ce contrôle est résumé dans un référé daté du 26 mai 2021 et publié le 14 septembre sur le site de la Cour. Les magistrats de la rue Cambon ont constaté que la nature de ces dépenses les exposait à des risques particuliers en termes d'image, d'économie et d'exemplarité. Bien que les missions du cabinet présentent indéniablement des caractéristiques propres, l'analyse des principales dépenses montre qu'il est nécessaire de mieux les encadrer pour en garantir la régularité et la transparence.

La Cour a ainsi relevé plusieurs risques tenant à certaines rémunérations ainsi qu'à la gestion des déclarations d'intérêts des membres du cabinet. Les délégations de signature, comme le contrôle budgétaire et comptable des dépenses, sont insuffisamment encadrées. Les frais de bouche et de transport peuvent être mieux contenus. Aussi, des indemnités sont versées aux fonctionnaires du bureau du cabinet dont les tâches administratives n'impliquent pas de sujétions spécifiques. Tout en prenant acte du début de remise en ordre intervenu dans le contexte de son enquête, la Cour formule au total cinq recommandations en lien avec les défaillances relevées.

\section{$\pi$ Budget des opérateurs}

\section{L'établissement de crédit SFIL (ex-société de financement local)}

La SFIL a été créé en 2013 dans le cadre du plan de résolution ordonnée du groupe Dexia et reconnu en tant que banque de développement par la Commission européenne. La SFIL était à l'origine détenue à $75 \%$ par l'État, $5 \%$ par la 
Banque postale et 20 \% par la Caisse des dépôts et consignations qui en a pris le total contrôle en septembre 2020. À partir des ressources empruntées sur les marchés par sa filiale Caffil (Caisse française de financement local), le groupe SFIL octroie des prêts aux collectivités locales, aux hôpitaux français et aux acheteurs d'exportations françaises. Fin 2020, le bilan du groupe s'élevait à $77 \mathrm{Md}$, pour un résultat net au titre de l'exercice de $44 \mathrm{M} €$ et un effectif de près de 400 salariés.

À l'issue de son contrôle au titre des exercices 2016-2019 et du premier semestre 2020, la Cour tire un bilan positif de l'activité du groupe sur la période visée. Dans son rapport d'observations définitives délibéré le 17 juin 2021 et rendu public le 14 septembre 2021 la Cour considère que la mission de la SFIL de désensibilisation des prêts sensibles est en large partie menée à bien, avec l'aide financière de l'État. Le groupe est par ailleurs devenu un acteur important du financement du secteur public local, en partenariat avec la Banque Postale. Enfin, le dispositif de soutien aux grands contrats à l'export mis en place en 2015 est désormais opérationnel.

Si la performance financière du groupe SFIL reste modeste, elle est cohérente avec son modèle économique. Selon la Cour, la SFIL appréhende correctement ses risques et respecte les ratios (notamment de solvabilité et de liquidité) qui lui sont applicables. La gestion des compétences demeure toutefois un enjeu pour le groupe. Enfin, des synergies restent encore à définir au sein du groupe Caisse des dépôts pour permettre des gains d'efficacité mutuels au sein des deux structures. La Cour formule au total sept recommandations.

\section{Comptabilité PUBliQUe}

\section{$\lambda$ Juridictions financières}

Vers une responsabilité unifiée des gestionnaires publics

Le projet de loi de finances 2022, en son article 41, habilite le Gouvernement à prendre par voie d'ordonnance toute mesure permettant d'harmoniser le régime juridictionnel de responsabilité des gestionnaires publics, passant notamment par une révision du code des juridictions financières.

L'objectif de la réforme est de définir un nouveau régime unifié de responsabilité applicable à l'ensemble des agents publics, comptables comme ordonnateurs, plus simple, plus lisible et plus juste. Il vise à sanctionner plus efficacement les fautes graves relatives à l'exécution des recettes et des dépenses ou à la gestion des biens provoquant des préjudices financiers significatifs. II s'agit de réserver l'office du juge aux cas d'une gravité avérée et non pas de sanctionner des fautes purement formelles. Dans ce cadre, la responsabilité personnelle et pécuniaire du comptable public sera abrogée et la Cour de discipline budgétaire et financière supprimée.

En complément de sanctions financières qui se matérialisent par des amendes pouvant aller jusqu'à six mois de rémunération, il est prévu $d^{\prime}$ 'instaurer la possibilité de prononcer une peine $d^{\prime}$ 'interdiction temporaire d'exercer les fonctions de comptable ou d'avoir la qualité d'ordonnateur. La nouvelle organisation juridictionnelle unifiée reposera sur trois niveaux. La Cour des comptes sera la seule juridiction en charge de la répression des fautes en première instance. Les magistrats des chambres régionales des comptes devraient néanmoins participer à l'instruction des dossiers et aux formations de jugement à la Cour. Une juridiction d'appel sera mise en place, présidée par le Premier président de la Cour des comptes et constituée de 4 membres de la Cour des comptes, 4 membres du Conseil d'État et de 2 personnalités qualifiées désignées pour leur expérience dans le domaine de la gestion publique. Les pourvois en cassation pourront être formés devant le Conseil d'État. En principe, la séparation fonctionnelle des ordonnateurs et des comptables et l'existence des contrôles de ces derniers ne sont pas remis en cause par la présente réforme.

En l'état, cette réforme qui doit entrer en vigueur au 1er janvier 2023 est ambitieuse mais ne résout pas l'intégralité des défauts de l'ancien système. Pour ne prendre qu'un exemple topique, les ministres et les élus ne se sont toujours pas concernés par le nouveau dispositif. Aussi, de grandes incertitudes entourent ce projet. Quelle sera la définition de la faute grave ? Quelle sera la définition du préjudice financier significatif? La réunion de ces deux notions ne portera-t-elle pas une atteinte trop importante à la régularité des opérations financières publiques? Quelle sera la place des responsabilités managériales et disciplinaires dans ce nouveau système ? Quelle sera l'articulation entre le nouveau système répressif de responsabilité financière avec les autres responsabilités juridictionnelles de type pénal ou administratif? Une responsabilité de type « civil » ayant pour objet la réparation du préjudice causé pourra-t-elle se cumuler avec le nouveau système? Quelle sera la place du cautionnement et du 
mécanisme assurantiel dans ce nouveau régime de responsabilité ? Des questions qui laissent le sujet de la responsabilité financière des gestionnaires publics encore plein de ressource et qui offrent au Gouvernement une importante marge de manœuvre pour perfectionner ce qui n'est encore qu'une esquisse - par définition inaboutie dans le projet de loi de finances pour 2022.

\section{FinANCES LOCALES}

\section{Projet de loi de finances pour 2022}

Après deux années mouvementées, le projet de loi de finances pour 2022 ne contient pas de réformes majeures. Cependant, ce texte n'est pas non plus totalement inintéressant. Comme tous les ans, quelques mesures d'habillage ont été prévues comme le doublement de la dotation dite "biodiversité » qui passe à $20 \mathrm{M} €$ au lieu de 10 .

Les contrats de relance et de transition écologique sont augmentés de $350 \mathrm{M} €$ par le biais de la dotation de soutien à l'investissement local (DSIL). En matière de subvention, la loi de finances maintient un niveau élevé avec 1,046 Md€ et $150 \mathrm{M} €$ respectivement pour la dotation d'équipement des territoires ruraux (DETR) et la dotation politique de la ville (DPV).

La dotation de soutien à l'investissement des départements doit également être revue afin de supprimer la part « péréquation » et gonfler d'autant la partie projet. Les départements sont particulièrement à l'honneur avec l'introduction d'une expérimentation concernant la recentralisation du RSA. Les régions, quant à elles, apparaissent délaissées avec une imputation de 50 millions du produit de TVA et une réforme de la péréquation toujours pas prête. Reste à voir si elle sera introduite par voie d'amendement.

\section{Libéralisation du financement participatif par la loi}

Les parlementaires ont définitivement adopté le 28 septembre la loi portant diverses dispositions d'adaptation au droit de l'Union européenne dans le domaine des transports, de l'environnement, de l'économie et des finances. Cette dernière contient un article 41 (numérotation peut-être non définitive) qui libéralise le recours au financement participatif (crowfunding) en l'autorisant pour désormais tous les services publics sauf missions de police et maintien de l'ordre.

En outre, "À titre expérimental et pour une durée de trois ans à compter du 1er janvier 2022, les collectivités territoriales et leurs établissements publics peuvent, à l'exclusion de toute exécution forcée de leurs créances, confier à un organisme public ou privé, après avis conforme de leur comptable public et par convention écrite, l'encaissement du revenu tiré d'un projet de financement participatif sous forme de titres de créance au profit de tout service public, à l'exception des missions de police et de maintien de l'ordre public».

\section{Perspectives d'emprunt élevées par la So- ciété de financement local (SFIL)}

Dans son rapport semestriel, la SFIL revient sur sa situation financière mais aussi sur les perspectives pour les mois à venir en matière $d^{\prime}$ emprunt et donc de dépenses d'investissement. Sur le premier semestre, la SFIL a développé sa franchise d'émetteur obligataire sur le segment des Agences Françaises en levant quasiment 1,8 Mds€. Au-delà de cet exemple typique, sa situation financière s'avère très saine. Les emprunteurs sont quasiment toutes des personnes morales de droit public. En outre, la SFIL couvre $100 \%$ du capital de la dette. Enfin, le risque de liquidités est surveillé de très près avec un financement du groupe majoritairement à long terme par émission de covered bonds.

\section{Les territoires urbains - Portrait financier - Édition $n^{\circ} 6$}

Dans cette nouvelle analyse de France Urbaine, cette association d'élus locaux s'évertue à relativiser la bonne santé financière des collectivités. Deux chiffres sont mis en exergue, d'une part la chute des dépenses d'équipement de - 17,8\%, et, d'autre part, le recul de l'épargne brute de $-13,8 \%$

Ce rappel de France urbaine est d'autant plus important que cette association représente en 2020 des territoires urbains réalisant 77,7 Mds€ de dépenses, soit une baisse de - 3,1\% par rapport à 2019 dont une diminution de - $16 \%$ en investissement.

Ce point de vue démontre toute l'hétérogénéité des situations locales. La situation des autorités organisatrices de mobilité est également compliquée et disparate. À cet égard, le versement mobilité a connu une baisse de 5,1\% et la billetterie de $-32 \%$.

\section{Les finances des collectivités locales en 2021}

Cette note de la DGCL était particulièrement attendue pour analyser la nature de la reprise tant annoncée du côté des collectivités territoriales 
après une année terrible de baisse de l'investissement. En termes de dépenses de fonctionnement, celles-ci augmentent de $+2,4 \%$ avec une hausse concernant surtout les achats et les charges externes.

Cependant, il ne faudrait pas croire que tout est revenu à la normale. L'épargne brute continue à diminuer avec une baisse de l'ordre de $-3,9 \%$ au budget primitif 2021 après une baisse de -10,8\% en 2020.

Les recettes d'investissement (FCTVA, subventions reçues et dotations à l'investissement) augmenteraient, pour leur part, plus fortement (+ 10,4\%) que les dépenses d'investissement.

\section{FINANCES SOCIALES}

\section{$\lambda$ Les conséquences de la crise sanitaire et le soutien à l'économie}

La prolongation des aides à l'apprentissage jusqu'au 30 juin 2022

Suite à ses rencontres avec les partenaires sociaux, le Premier Ministre a annoncé le 6 septembre, devant le Conseil national de l'Industrie, la prolongation jusqu'au 30 juin 2022 des aides à l'apprentissage qui permettent une prise en charge jusqu'à $8000 €$ la première année (pour un apprenti de 21 à 25 ans) des charges salariales. L'effet incitatif de cette aide, qui peut conduire à couvrir jusqu'à $80 \%$ du salaire, a été spectaculaire puisque 526000 contrats d'apprentissage ont été recensés en 2020 (+ $42 \%$ par rapport à l'année précédente).

Le rapport de la Cour des Comptes sur le bilan opérationnel de l'utilisation des dépenses publiques pendant la crise

La Cour des Comptes a rendu le 9 septembre le rapport demandé par la commission des finances de l'Assemblée Nationale sur le bilan opérationnel de l'utilisation des dépenses publiques pendant la crise. La Cour fait le constat d'une augmentation des dépenses publiques - en comptabilité nationale - de 96,4 Md€, dont $86 \%$ seraient imputables à la crise ((83 Md€). Les dépenses de l'État ont progressé de 52,9 Md€. L'essentiel est représenté par la mission «Plan d'urgence pour faire face à la crise sanitaire »: prise en charge de l'activité partielle (17,8 Md€), Fonds de solidarité (11,8 Md€), renforcement des participations financières dans les secteurs stratégiques (8,3 Md€), compensation des exo- nérations de charges sociales (3,9 Md€); s'y ajoutent les aides au revenu des ménages, les aides à l'emploi, des concours aux collectivités territoriales. Les dépenses nettes des régimes obligatoires de base et du FSV ont progressé de 27,1 Md€, là aussi quasi exclusivement imputables à la crise et concentrées sur l'Assurance maladie (dont 1,4 Md€ au titre du « Ségur de la Santé - qui représentera sur plusieurs années 22Md€ - voir Repères d'août) ; il faut compléter ce montant par 14,6 MdE à la charge de I'UNEDIC, principalement au titre du chômage partiel. En revanche les dépenses des collectivités territoriales auront diminué de 2,3 Md€ en 2020, du fait notamment d'une contraction de l'investissement. La Cour relève que les conséquences de la crise sur les finances publiques pourraient être d'un montant équivalent en 2021, et se prolonger au-delà, du fait du Ségur de la santé, de la montée en charge du plan de relance ou de la mise en jeu des garanties de prêts par l'État. La Cour considère que les objectifs poursuivis -notamment pour le soutien aux entreprises- ont été globalement atteints à court terme, grâce à des dispositifs souvent plus favorables que chez nos partenaires (Fonds de solidarité, PGE...), mais avec les risques d'effets d'aubaine et de fraude significatifs, en particulier pour le Fonds de solidarité et l'activité partielle. La Cour ne va pas sans soulever la question de l'efficience de ces aides sur le long terme dans la mesure où elles ont conduit à aggraver sensiblement l'endettement des entreprises. S'agissant de l'objectif du soutien à l'emploi, les dispositifs ont permis de contenir efficacement le chômage, sauf pour les salariés en contrats précaires. Enfin le revenu des ménages a globalement été bien préservé puisqu'il a même augmenté de $1 \%$ en 2020, alors que le PIB reculait de $8 \%$. La Cour formule enfin une série de 6 recommandations visant notamment à renforcer les contrôles pour éviter le cumul des aides et à organiser les dispositifs de sortie de crise.

\section{Le PLF 2022 consacre l'extinction progres- sive des dispositifs exceptionnels de sou- tien économique}

Les crédits de la mission "Plan d'urgence » du PLF 2022 seront pratiquement ramenés à zéro, puisque ne sont inscrits que $0,2 \mathrm{Md}$, après une mobilisation à ce titre de près de $80 \mathrm{MdE}$ sur 2020 et 2021 ; cela consacre l'extinction des 3 « piliers » du dispositif de soutien à l'économie qui ont été mis en place pour soutenir le tissu économique pendant la crise : I'activité partielle, dont le niveau de prise en charge a été diminué progres- 
sivement pendant l'été et qui doit s'éteindre le 31 octobre ; le premier volet du Fonds de solidarité qui s'arrête le 30 septembre, certaines aides pour les secteurs qui restent encore fortement impactés par la crise étant toutefois prolongées ; la prise en charge des exonérations et aides au paiement de cotisations sociales qui s'est arrêtée le 31 août dernier. Des reports permettront de liquider les éventuels restes à payer en 2022, mais selon le Gouvernement, aucun besoin n'est identifié pour 2022 au titre de l'activité partielle et du Fonds de solidarité. Par ailleurs le dispositif de prêts garantis par l'État a vu sa date limite d'octroi prolongée jusqu'au 31 décembre 2021, la charge budgétaire de ce mécanisme ne pouvant être constatée qu'au vu des défaillances de remboursement des prêts à la période d'échéance. Mais, sur ce point, une note du Conseil d'analyse économique sur la situation financière des TPEPME attire l'attention sur le fait que si la situation financière de ces entreprises est restée correcte grâce au soutien massif des pouvoirs publics, 15 à $25 \%$ de celles qui ont souscrit un PGE pourraient avoir des difficultés pour rembourser la première échéance au printemps 2022, d'autant que le brutal renchérissement des tarifs de l'énergie est venu compliquer les perspectives de la reprise. Par ailleurs, le grand absent de ce PLF 2022 est le "Revenu universel d'engagement », annoncé par le Président de la République en juillet dernier, dont la mise au point paraît s'avérer très problématique.

\section{त Situation des comptes sociaux}

Le rapport de la Commission des comptes de la Sécurité sociale de septembre 2021

La Commission des comptes de la SS a présenté son rapport pour les exercices 2021 et 2022. Pour 2021, elle anticipe un déficit global du Régime général et du FSV de -34,5 Md€ (contre - 38,7 Md€ en 2020), inférieur à ce que la Commission prévoyait au mois de juin (- 38,4 Md€), les autres régimes obligatoires de base présentant un déficit global de - 0,2 Md€. Comme l'an dernier, le déficit est essentiellement porté par l'Assurance maladie (- 29,4 Md€, montant pratiquement équivalent à celui de 2020); les branches Accidents du travail et Famille renoueraient avec les excédents (respectivement 0,6 Md€ et 1,2 Md€), la Vieillesse et le FSV seraient au même niveau que l'an dernier (respectivement - 3,7 Md€ et - 2,4 Md€) et la branche Autonomie enregistrerait un déficit de - 0,9 Mdv. Cette amélioration du solde global est imputable à un dynamisme beaucoup plus important qu'attendu des re- cettes assises sur la masse salariale du secteur privé (+ 6,2\%), tel que l'estiment les nouvelles hypothèses économiques de septembre 2021, cela malgré un surcroît de dépenses de santé dû à la crise. En effet, les dépenses entrant dans le champ de I'ONDAM auront encore progressé de $+7,4 \%$ par rapport à l'année 2020 (où elles avaient déjà augmenté de $+9,4 \%$ ), ce dépassement par rapport aux prévisions de la LFSS de 2021 étant principalement imputable aux charges liées aux tests et aux campagnes de vaccination.

Pour 2022, avant toute prise en compte de mesures nouvelles du PLFSS 2022 (cf infra), le déficit tendanciel du Régime général et du FSV serait ramené à - 22,1 Md€, grâce à la poursuite de la progression des recettes $(+3,9 \%)$ et au freinage des dépenses $(+0,7 \%)$ du fait de moindres besoins au titre de la crise sanitaire. Avant mesures nouvelles, la progression des dépenses entrant dans le champ de l'ONDAM serait de 0,7 \%, la forte décrue des charges liées à la lutte contre l'épidémie (une provision de 4,9 Md€ est prévue pour la vaccination et pour les tests) étant en partie neutralisée par l'effet des premières mesures prises au titre du Ségur de la santé. De ce fait, le déficit tendanciel de l'Assurance maladie serait ramené à - 20,8 Md€, celui de la Vieillesse à -2,7 Md€ et du FSV à - 1,5 Md€, les Accidents du travail et la Famille voyant leurs excédents portés à 1,4 Md€ et 2,7 Md€.

\section{Loi de financement de la Sécurité Sociale}

\section{La présentation du PLFSS pour 2022}

Les ministres des solidarités et de la santé et de l'économie et des finances ont présenté le 24 septembre le projet de loi de financement de la Sécurité sociale pour 2022. Le déficit prévisionnel du régime général et du Fonds de solidarité vieillesse (FSV) a été finalement arrêté à 21,6 Md€ (- 22,6 Md€ si on prend en compte l'ensemble des régimes obligatoires de base). Le déficit de la branche Maladie serait de - 19,7 Md€, celui de la branche retraite de - 2,5 Md€, celui de la branche Autonomie de - 0,9 Md€, les autres branches étant en excédent (+ 1,3 Md€ pour les Accidents du travail et $+1,7 \mathrm{Md} €$ pour la Famille). Pour les exercices suivants, la réduction du déficit se poursuivrait en 2023 (- 14,6 Md€), mais plafonnerait en 2024 et 2025 autour de - 13 Md€. Si le déficit de l'Assurance maladie se maintiendrait autour de - $15 \mathrm{Md€}$, fortement impacté par la montée en charge du Ségur de la Santé, le déficit de la Vieillesse (y compris FSV) progresserait de façon inquiétante jusqu'à - 8Md€€ en 2025 ; 
le déficit global ne serait contenu que par une prévision d'excédents importants sur les autres branches, notamment la Famille. S'agissant du financement du déficit, le PLFSS confirme la poursuite des transferts de dette à la CADES, à hauteur de 40 Md€ en 2022 ; de ce fait, l'ACOSS va réduire son financement externe en 2022, et son plafond d'emprunt sera ramené à $65 \mathrm{Md}$, après avoir atteint le niveau historique de $95 \mathrm{Md} €$ en mai 2020. Le dossier de presse comporte une présentation détaillée de la montée en charge du Ségur de la Santé et de la mise en œuvre de la politique d'autonomie (à défaut du projet de loi sur la dépendance qui ne verra pas le jour sous ce quinquennat). II revient aussi sur une inflexion importante de la politique de dépense de santé dans ce PLFSS, qui renoue avec une croissance significative des dépenses de médicaments et de produits de santé (+1 Md€ en 2022) alors que celles-ci servaient traditionnellement d'ajustement pour le bouclage des ONDAM. Ceci fait suite aux décisions du Conseil stratégique des industries de santé de juin 2021(voir Repères de juin 2021), qui a fixé l'objectif de faire de la France la première nation européenne innovante et souveraine en santé en 2030, cela après la polémique qui s'est fait jour sur le décrochage de la France dans ce secteur, illustrée notamment par l'échec de Sanofi à produire un vaccin contre la COVID-19 avant la fin de 2021.

\section{$\lambda$ Dépenses de santé/Hôpital/ Assurance maladie}

\section{Le reste à charge des ménages en matière de santé continue de diminuer}

La publication annuelle de la DRESS sur les dépenses de santé, paradoxalement, fait apparaître que la progression de la consommation de soins et de biens médicaux (CSBM) a été, en 2020, la plus faible de ces dernières années (+ 0,4\%). Cela résulte de la crise sanitaire, qui certes a conduit à l'augmentation des soins hospitaliers publics et des dépenses d'analyses de laboratoire, mais en sens inverse les dépenses de soins de ville se sont contractées, particulièrement pour l'activité des médecins spécialistes et des dentistes ainsi que pour la consommation des médicaments en ambulatoire, de même que les cures thermales se sont effondrées. Cette dépense de CSBM est prise en charge à $80 \%$ par la Sécurité sociale, en progression de 1,9 points par rapport à 2019 du fait de la crise sanitaire, la part des organismes complémentaires santé baisse à 12,3\% en raison de la décrue des soins de ville, celle de l'État (Aide à la complémentaire santé solidaire, $\mathrm{AME}$ ) reste stable à $1,4 \%$; de ce fait le reste à charge des ménages tombe à 6,5\% contre 7,2 \% en 2019, du fait de la baisse de la consommation où le reste à charge est traditionnellement élevé (ex. dentistes)

\section{Un rapport de la Cour des Comptes sur les soins critiques}

Un rapport de la Cour des comptes, réalisé à la demande de la commission des affaires sociales du Sénat et rendu public le 22 septembre fait apparaître que la saturation des soins critiques, qui a explosé pendant les premières vagues de la pandémie et qui n'a pu être palliée que par la déprogrammation d'interventions dont les conséquences à long terme devront être évaluées, a été le révélateur de difficultés structurelles anciennes. La capacité en soins critiques (stabilisée autour de 5000 lits) n'a pas suivi l'évolution démographique et le vieillissement de la population, et le caractère mal financé de l'activité de soins critiques n'a pas incité les gestionnaires à augmenter les capacités. Par ailleurs l'offre en soins critiques est très hétérogène selon les régions. La Cour formule douze recommandations, dont les plus notables sont l'augmentation du nombre de postes ouverts en anesthésie-réanimation et la révision du mode de financement des soins critiques.

\section{$\pi$ Retraites}

\section{La relance mort-née de la réforme des re- traites}

Les Échos du 7 septembre faisaient état de l'intention du Président de la république de relancer a minima la réforme des retraites, en la limitant à la suppression des régimes spéciaux et à la revalorisation à $1000 €$ du minimum de pensions, sans remise à plat du mode de financement des régimes, ces deux réformes pouvant s'opérer par décret. Le Premier ministre s'était pourtant montré extrêmement prudent à l'issue de ses rencontres avec les partenaires sociaux le 2 septembre. Cette idée semble avoir été définitivement enterrée par le Chef de l'État lui-même lors de son déplacement en Provence le 10 septembre. Entretemps le débat sur le fond de de la réforme avait été relancé par un rapport, émanant de I'Institut Molinari et de Croissance, plus en faveur de la mise en place de la capitalisation pour tous en complément des régimes de répartition. 
La perspective de revalorisation des retraites complémentaires de 1,5\%

Selon des informations parues dans les Échos du 20 septembre, le Conseil d'administration de I'AGIRC-ARRCO pourrait revaloriser les retraites complémentaires de 1,5\%, en application de l'accord intervenu entre le patronat et deux syndicats en juillet (voir Repères de juillet 2021). Cette revalorisation doit se situer en deçà de 0,5 points de l'inflation attendue; celle-ci s'étant élevée à $2,2 \%$ en août, la revalorisation devrait donc être comprise entre 1,5 et $1,7 \%$.

\section{$\lambda$ Formation professionnelle}

Le Premier Ministre annonce un plan d'investissement en compétences de 1,4 Md€

Le Premier Ministre a dévoilé à Chatellerault, le 27 septembre, le contenu du " plan d'investissement en compétences ». Destiné à réduire les tensions en recrutement qui se sont fait jour dans plusieurs secteurs (hôtellerie restauration, BTP, santé et grand âge, transports et logistique...), le plan comporte 3 volets : la formation des salariés et des demandeurs d'emploi pour répondre aux besoins immédiats des entreprises (600 M€), la facilitation des transitions professionnelles, qui passe par la relance du dispositif «Transco » créé au début de 2021, mais dont les résultats ont été décevants (560 M€) et l'orientation vers des contrats de professionnalisation des chômeurs de longue durée (240 M€). Ce plan sera financé par redéploiement sur les reliquats du Plan d'urgence.

\section{入 Solidarité/Lutte contre la pauvreté/ Revenu universel}

Une enquête de I'INSEE éclaire le niveau de la pauvreté en France en 2019

Une enquête de I'INSEE publiée le 1er septembre apporte un éclairage sur la portée du concept de pauvreté et son niveau en France en 2019. Le concept de pauvreté monétaire, défini comme un niveau de revenu inférieur à $60 \%$ du revenu médian, est bien connu. Le concept de "pauvreté en conditions de vie » est fondé sur les conséquences de la faiblesse des revenus : privations, impossibilité d'acquérir ou de consommer certains biens ou d'honorer certaines dépenses obligatoires. II existe plusieurs indicateurs de cette pauvreté "non monétaire ", qui ne se recouvrent pas avec ceux de la pauvreté monétaire. L'INSEE constate une baisse tendancielle de la pauvreté non monétaire (entre $11 \%$ et $13 \%$ de la popula- tion selon les indicateurs pris en considération). Au sens le plus large, I'INSEE relève que $21 \%$ des français (soit un cinquième de la population totale) se trouvent en situation de pauvreté monétaire ou de privation matérielle et sociale. Les analyses sur la pauvreté peuvent être complétées par la note de France Stratégie sur les dépenses "préengagées « des ménages, qui représenteraient à présent près d'un tiers du total de leurs dépenses en 2017.

L'enquête annuelle de la DREES sur les titulaires de minima sociaux

La publication annuelle de la DREES sur les titulaires de minima sociaux fait apparaître une légère progression en $2019(+1,2 \%)$ du nombre de titulaires de minima sociaux (4,3 millions, 6,9 millions si on inclut tous les ayant-droit, soit $10 \%$ de la population). Les minima sociaux et autres prestations non contributives (ex. aides au logement, garantie jeune, prestations familiales, prestations familiales et prime d'activité) contribueraient à réduire de 7,5 points le taux de pauvreté en France, qui s'établit à $14,8 \%$ de la population métropolitaine (contre 22,3\% sans la redistribution).

\section{FISCALITÉ ET PROCÉDURES FISCALES}

\section{$\lambda$ Contrôle fiscal}

La publication du rapport d'activité de la DGFIP pour 2020

La DGFiP a présenté son rapport d'activité après une année 2020 bien particulière, marquée par la crise sanitaire. Ce rapport confirme une baisse des recettes liées aux contrôles fiscaux en raison des circonstances exceptionnelles. Au total, I'État a recouvré $7,8 \mathrm{Mds} €$ en 2020, contre 11 Mds€€ en 2019, un montant néanmoins équivalent à celui de 2018. Le montant total des redressements établis à la suite des contrôles fiscaux a atteint 10,2 Mds€, au lieu de 13,5 Mds€ en 2019. Ils ont principalement concerné l'IS (2 Mds€) et les remboursements de crédits de TVA (1,7 Mds€). En raison de la crise liée à la Covid-19, I'administration fiscale a été contrainte de s'adapter aux circonstances. Durant le premier confinement, les services fiscaux se sont concentrés sur les contrôles sur pièces au détriment de ceux effectués sur place, et ils ont ciblé leurs missions de contrôle sur les dossiers patrimoniaux à forts en- 
jeux. Ainsi, ce sont seulement 104586 contrôles sur pièces des professionnels qui ont eu lieu en 2020, contre 137095 en 2019. Et ce sont seulement 17483 contrôles qui se sont déroulés dans les locaux des entreprises, contre 35545 en 2019. Au sens large, toutes procédures fiscales confondues, ce sont un peu plus de 272000 contrôles fiscaux qui ont été menés auprès des professionnels (374 000 en 2019). Afin de faire face à la fraude fiscale, la DGFiP s'appuie enfin de plus en plus sur l'intelligence artificielle et le data mining afin de moderniser les techniques d'analyse. En 2020, 794 M€ de droits et pénalités ont été rappelés grâce au data mining. Près d'un contrôle sur trois a été ciblé par l'intelligence artificielle (contre seulement $22 \%$ en 2019). Enfin, la part des contrôles qui se sont conclus par une acceptation du contribuable est également en forte hausse puisqu'elle s'est établie à près de $37 \%$, au lieu de $24 \%$ en 2019. Et plus de 31000 dossiers ont fait l'objet d'une régularisation en cours de contrôle. Depuis octobre 2020, les professionnels peuvent désormais régler, en ligne, leurs dettes fiscales en phase de recouvrement forcé.

\section{$\lambda$ Impôt sur le capital}

\section{Des précisions sur l'imposition foncière des mobil-home}

Dans le cadre d'une réponse ministérielle à la question écrite du député Houlié (v. QE n 37711, JOAN du 7 sept. 2021) le Gouvernement vient d'apporter des précisions concernant l'assujettissement à la TFPB des propriétaires de terrain privé avec mobil-home, dites résidences mobiles de loisirs, non fixées au sol à perpétuelle demeure. Le régime fiscal applicable à ces résidences (C. Urb., art. R. 111-41) est conditionné par l'examen de la situation de fait propre à chaque installation. S'agit-il d'une construction démontable ou transportable, destinée à une occupation temporaire ou saisonnière à usage de loisirs (C. Urb., art. R. 111-37) ou d'une construction qui aurait perdu ses moyens de mobilité ? Ainsi, un mobil-home qui a perdu ses moyens de mobilité, installé dans un terrain privé, et qui n'a pas vocation à être déplacé régulièrement et facilement est alors susceptible d'être assimilé à une construction fixée au sol à perpétuelle demeure et imposé à la TFPB (CE, 28 déc. 2005, Société Foncicast, Req. n 266558 ; v. notre étude, L'essentiel du droit de la fiscalité locale, Lextenso, 2020). Quant au terrain, en principe imposable à la TFPNB (CGI, art. 1381), il devient soumis à la TFPB dès lors qu'il devient un terrain non cultivé, employé à un usage commercial ou industriel.
La responsabilité du notaire et l'exonération de plus-value immobilière sur résidence principale

La première chambre civile de la Cour de cassation vient de rappeler que dans le cadre d'un litige portant sur la mise en œuvre de l'exonération de plus-value immobilière le notaire qui a connaissance avant la vente de la fausseté de l'affirmation selon laquelle le bien constituait au jour de la vente la résidence principale du client, engage sa responsabilité au sens de l'article 1382 du Code civil (Cass., Civ. $1^{\text {re }}, 22$ sept. 2021, Pourvoi $\left.n^{\circ} 19-23.506\right)$.

\section{$\lambda$ Politique fiscale}

Vers une réforme de I'IFER sur les antennes mobiles en loi de finances pour 2022 (2) ?

Le législateur avait prévu la remise au Parlement d'un rapport relatif à l'imposition forfaitaire sur les entreprises de réseaux (IFER) appliquée aux stations radioélectriques, à savoir les antennes relais de téléphonie mobile (art. 129 L. n 2019 1479 du 28 déc. 2019 de finances pour 2020). Dans un rapport demeuré confidentiel, I'Inspection générale des finances (IGF) a relancé le débat autour d'une réforme fiscale qui inquiète les associations d'élus locaux après la réforme de la taxe d'habitation et des impôts de production (v. Repères, août 2021). La réforme envisagée a été abandonnée par le Gouvernement, ce qui a été confirmé par le ministre chargé des comptes publics, qui a souligné : «nous n'avons pas inscrit la réforme de l'IFER dans le PLF 2022 car c'est un sujet complexe qui mérite du temps et qui n'a pas de caractère d'urgence ». Cela a été confirmé par le rapporteur général du Budget à l'Assemblée nationale. Ces annonces ne devraient toutefois pas décourager certains députés de la majorité parlementaire de proposer des amendements en ce sens. À suivre...

\section{Le décret relatif à la suppression des taxes à faible rendement budgétaire}

En application de dispositions prises par le législateur (art. 64 et 121, L. n²020-1721 du 29 déc. 2020 de finances pour 2021) qui a procédé à la suppression de quatorze taxes à rendement budgétaire faible (taxes funéraires, droits d'enregistrement des actes constatant la cession à titre onéreux d'un fonds agricole), le Premier ministre, après avis de la section des finances du Conseil d'État, a pris un décret relatif à la suppression de taxes à faible rendement à compter de 2021 (Décr. n²021-984 du 26 juillet 2021, JORF 28 
juillet 2021) par rapport à des abrogations de références au niveau réglementaire (taxes spéciales sur la pornographie; la composante de la TGAP portant sur les lubrifiants, les huiles et les produits lubrifiants ; les prélèvements progressif et complémentaire sur le produit brut des jeux dans les casinos installés à bord des navires de commerce transporteurs de passagers battant pavillon français; le droit sur les déclarations et notifications de produits du tabac ; la taxe à l'essieu sur les véhicules immatriculés dans un État étranger taxant les véhicules immatriculés en France). Si la démarche vise à simplifier l'état du droit, à alléger la pression fiscale et les formalités déclaratives des entreprises, elle vise surtout à réduire les coûts budgétaires de recouvrement dans le sens des recommandations formulées par la Cour des comptes (C. comptes, référé sur la suppression des taxes à faible rendement, 3 déc. 2018, n S2018-3303 - V. Repères, févr. 2019). II s'agit d'un programme pluriannuel de réduction du nombre de taxes à faible rendement engagé par le législateur (art. 26 L. n²018-1317 du 28 déc. 2018 de finances pour 2019), qui a supprimé (taxes sur les farines, huiles végétales, céréales) ou modifié (taxe de balayage), certaines taxes. Cette démarche a été poursuivie par le législateur en 2020 (art. 21 L. n²019-1479 du 28 déc. 2019 de finances pour 2020) par la suppression de certaines petites taxes à faible rendement budgétaire (taxe sur les plus-values de cession de logements sociaux, la taxe sur les activités commerciales non salariées à durée saisonnière, la contribution perçue au profit de I'INPES, la taxe sur les produits du vapotage, la taxe sur les premières ventes de dispositifs médicaux, du droit fixe dû par les entreprises ferroviaires au profit de I'ART, la taxe sur les voyageurs de commerce, la taxe annuelle sur la détention de véhicules polluants, et du droit de sécurité dû par les entreprises ferroviaires).

\section{$\lambda$ Impôt sur les sociétés}

La fusion avisée d'une date d'effet rétroactif : le Conseil d'État valide la doctrine administrative

Le Conseil d'État (CE, $8^{e}$ et $3^{e}$ ch. r., 13 sept. 2021, SAS Adis et SCI Rocca, Req. $n^{\circ} 451564$ ) a décidé de rejeter la requête en annulation pour excès de pouvoir de la décision du 24 février 2021 par laquelle la DGFIP a refusé d'abroger les $\S 80, \S 90$ et $\S 110$ des commentaires BOFiP-impôts (BOI-ISFUS-40-10-20) par lesquels il a fait connaître son interprétation de la loi fiscale en ce qui concerne les conséquences à tirer, en matière $d$ 'imposition des bénéfices des sociétés, des clauses de fusion avisant une date d'effet rétroactif.

\section{TVA}

L'ordonnance relative à la généralisation de la facture électronique

Après que la DGFIP ait soumis à consultation publique du 13 août au 13 octobre 2021 ses commentaires de la transposition du paquet TVA dit « commerce électronique » (v. Repères août 2021), le Gouvernement vient de prendre une ordonnance relative à la généralisation de la facturation électronique, jusqu'alors facultative, dans les transactions entre assujetties à la TVA et à la transmission des données de transaction (Ord. $n^{\circ}$ 2021-1190 du 15 sept. 2021, JORF n 0216, 16 sept. 2021) sur habilitation donnée par le législateur pour ce faire (art. 195 L. n ${ }^{\circ} 2020-1721$ du 29 déc. 2020 de finances pour 2021). Un calendrier graduel est prévu pour la mise en œuvre de cette nouvelle obligation d'émission des factures sous forme électronique (CGI, art. 289-VI) : 1er janvier 2024 pour les grandes entreprises ; 1 er janvier 2025 pour les entreprises de taille intermédiaire ; 1er janvier 2026 pour les PME et les TPE.

La déductibilité de la TVA dans le cadre d'une opération patrimoniale d'une holding

Dans cette affaire, une holding a exposé des frais d'avocat et d'instance à l'occasion d'un litige dans le cadre d'une opération patrimoniale. À la suite d'une vérification de comptabilité de celle-ci, I'administration fiscale a remis en cause la déduction de la TVA ayant grevé ces frais d'avocat et d'instance. Le Conseil d'État vient d'annuler l'arrêt de la CAA de Nancy du 8 avril 2020 estimant que ces frais d'avocat et d'instance se rattachaient à une opération à caractère purement patrimonial car il ne résulte pas de l'instruction qu'au-delà de son statut de propriétaire de titres, elle se serait immiscée dans la gestion desdites sociétés et leur aurait fourni des prestations de services soumises à la TVA (CE, $3^{\mathrm{e}}$ et $8^{\mathrm{e}} \mathrm{ch}$. r., 28 sept. 2021, SARL Saint-Exupéry Holding, Req. $n^{\circ}$ 440987).

\section{$\pi$ Politique fiscale}

Les dispositions fiscales du projet de loi de finances pour 2022

Le projet de loi de finances pour 2022 a été présenté en conseil des ministres du 22 septembre par le ministre de l'économie et des finances et le ministre chargé des comptes publics. Depuis 2017, les prélèvements obligatoires ont été réduits de 50 Mds€ selon le Gouvernement, avec 
pour 2022, un taux de prélèvements obligatoires établi à 43,5 \% du PIB (contre 45,1\% en 2017). On retrouve notamment la poursuite de la suppression de la TH sur la résidence principale pour les $20 \%$ de foyers fiscaux encore concernés par celle-ci (exonération à hauteur de $65 \%$ ) avant sa suppression définitive en 2023 et la baisse du taux normal d'IS établi désormais à $25 \%$. Le Gouvernement estime avoir beaucoup fait depuis 2017 en matière d'allègements fiscaux (réforme de I'IFI, du PFU, du PAS, etc.) et semble opter pour la stabilité de la législation fiscale dans le cadre du dernier projet de loi de finances initiale du quinquennat comme en témoigne l'abandon de la réforme de I'IFER. Par ailleurs, il faut souligner la très bonne "surprise » sur le niveau de recettes fiscales en 2021 puisque selon le Gouvernement les impôts et taxes devraient générer 19,6 Mds€ de recettes fiscales supplémentaires par rapport à que ce qui était prévu en loi de finances rectificative adoptée par le Parlement juste avant la trêve estivale (L. $n^{\circ} 2021-953$ du 19 juillet 2021 de finances rectificative pour 2021) et non soumise au contrôle de constitutionnalité, notamment de la sincérité de ces prévisions.

Parmi les mesures fiscales intéressant les particuliers, en matière $d^{\prime} I R$, on retrouve l'indexation sur l'inflation (évolution de l'indice des prix à la consommation hors tabac, soit 1,4\%) du barème de I'IR pour les revenus 2020 et des grilles de taux par défaut du PAS (PLF 2022, art. 2) ; le renforcement de la sécurité juridique des prestations de services éligibles au crédit d'impôt en faveur des services à la personne (art. 3 du PLF 2022 qui inscrit dans la loi les commentaires administratifs annulés par CE, 8e et 3e ch. r., 30 nov. 2020, Société Les jardins d'Iroise de Auch, Req. n442046 v. Repères, févr. 2021) ; la prorogation de l'abattement sur les plus-values réalisées par un dirigeant de PME partant à la retraite (PLF 2022, art. 5) ; la non indexation sur l'inflation de la contribution à l'audiovisuel public fixée à $138 €$ en métropole et $88 €$ en outre-mer (PLF 2022, art. 16). Parmi les mesures fiscales intéressant les entreprises, on retrouve l'allongement des délais d'option pour les régimes d'imposition à l'IR des entrepreneurs individuels (PLF 2022, art. 4) ; le renforcement du crédit d'impôt pour la formation des chefs d'entreprise et l'aménagement du régime d'exonération de plus-value professionnelle pour départ à la retraite (PLF 2022, art. 5) ; la faculté d'admettre à titre temporaire en déduction l'amortissement pour les fonds commerciaux acquis entre le 1er janvier 2022 et le 31 décembre 2023 (PLF 2022, art. 6) ; la mise en conformité avec le droit de l'UE des retenues à la source applicables aux sociétés non résidentes (PLF 2022, art. 7) suite aux arrêts du Conseil d'État par lesquels certains dispositifs de retenue à la source ont été considérés comme contraires aux principes de liberté de circulation des capitaux et de libre prestation de services garantis par le TFUE (CE, 8e et $3^{\mathrm{e}}$ ch. r., 11 mai 2021, Société UBS Asset Management Life Ltd, Req. $n^{\circ} 438135$; CE, 8 e et 3 e ch. r., 09 sept. 2020, SAS Damolin Etrechy, Req. $n^{\circ} 434364$; CE, 8e et 3e ch. r., 22 nov. 2019, SAEM de gestion du Port Vauban, Req. $n^{\circ} 423698$ ) ; la mise en conformité avec le droit de I'UE de certaines règles en matière de TVA (PLF 2022, art. 9) ; la suppression de six dépenses fiscales jugées inefficaces par le Gouvernement (PLF 2022, art. 10) comme la réduction d'IR au titre des dépenses réalisées sur certains espaces naturels en vue du maintien et de la protection du patrimoine naturel (CGI, art. 199 octovicies) ; I'exonération d'IR des lots d'obligations et primes de remboursement attachées à des emprunts négociables émis avant le 1er janvier 1992 ; l'exonération d'IR des intérêts des sommes inscrites sur un compte épargne d'assurance pour la forêt (CEAF) ouverts jusqu'au 31 décembre 2013 (CGI, art. 157-23) ; I'exonération d'IR ou d'IS, plafonnée à 61000 € de bénéfice, pour les entreprises qui exercent une activité en ZFU (CGI, art. 44 octies) ; I'exonération d'IS des bénéfices réalisés, au cours des 24 mois suivant leur création, par les sociétés créées entre

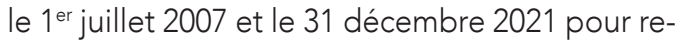
prendre une entreprise ou des établissements industriels en difficulté (CGI, art. 44 septies) ; I'exonération, sur agrément, des bénéfices réinvestis dans l'entreprise pour les sociétés de recherche et d'exploitation minière dans les départements d'outre-mer (CGI, art. 1655 bis) ; Par ailleurs, le renouvellement de la décision d'autorisation du régime de taxation différenciée à l'octroi de mer adoptée par le Conseil de l'UE (décision (UE) n²021-991 du 7 juin 2021), qui permet la poursuite de ce dispositif jusqu'au 31 décembre 2027 (PLF 2022, art. 31) ; Enfin, la création d'une taxe affectée à l'Autorité des relations sociales des plateformes d'emploi (ARPE) qui ciblera les plateformes de VTC et de livraison et sera affectée à un organisme chargé d'organiser les élections professionnelles du secteur (1,5 et $2 \mathrm{M} €$ par an ; PLF 2022, art. 32).

\section{Management PUblic}

\section{$\pi$ Gestion publique}

\section{L'évaluation des politiques publiques}

Le Cercle de la Réforme de l'État a publié le 13 septembre 2021 une note sur l'évaluation des politiques publiques « au service de la réforme 
de l'État : réconcilier la connaissance et l'action, opérationnaliser l'évaluation". Ce document d'une douzaine de pages vise à ce que l'évaluation « ne soit pas perçue comme une activité d'experts [mais qu'elle] réponde effectivement aux attentes de la société ». Parmi les questions abordées figurent:

- la complexité de la prise de décision dans un contexte d'incertitude ;

- la situation française où l'évaluation a certes bien progressé (en méthodologie et en pratique) mais ne se trouve toujours pas «à maturité »;

- I'obligation de l'évaluation dans le cycle de la décision publique.

\section{$\pi$ Fonction publique}

\section{La formation à la laïcité des enseignants}

Un arrêté publié le 12 septembre 2021 définit « le cahier des charges relatif au continuum de formation obligatoire des personnels enseignants et d'éducation concernant la laïcité et les valeurs de la République».

L'objectif de la formation est de connaître :

- le principe de laïcité et ses dimensions juridiques, historiques et philosophiques ;

- ce que sont les «faits religieux»;

- les grands principes qui régissent le cadre juridique de la laïcité à l'école ;

- les finalités, les valeurs et les principes fondateurs du service public de l'éducation.

Parmi les autres dispositions figurant dans le texte, il convient de préciser que:

- cette formation s'adresse à tous les étudiants engagés dans un master des métiers de l'enseignement, de l'éducation et de la formation MEEF ;

- les fonctionnaires stagiaires et les néo-titulaires feront l'objet d'un accompagnement spécifique ;

- la formation s'articule avec les priorités éducatives ministérielles énoncées dans le schéma directeur de la formation continue, notamment pour les personnels enseignants et d'éducation.

\section{La baisse de la sélectivité dans les concours de la fonction publique}

Une note publiée jeudi 16 septembre 2021 par la Direction générale de l'administration et de la fonction publique (DGAFP) confirme la tendance observée depuis plusieurs années d'une baisse de la sélectivité dans les concours de la fonction publique d'État. L'analyse porte sur les recrutements effectués en 2019. On note ainsi que :

- plus de six recrutés sur dix sont des femmes en 2019;

- moins d'une personne sur deux (47\%) inscrite à un recrutement s'est présentée aux premières épreuves ;

- la proportion de candidats présents à la première épreuve est plus faible pour les recrutements externes (40\%) que pour les recrutements internes (51\%);

- a sélectivité des recrutements dans la fonction publique de l'État s'élève à 6,6 présents pour un admis sur la liste principale en 2019 ;

- cette sélectivité est plus faible pour les recrutements externes $(6,2)$ que les recrutements internes $(7,8)$;

- plus d'un quart (26\%) des recrutés aux concours externes ou uniques ont un niveau d'études supérieur à celui exigé lors de l'inscription (par exemple, pour les concours de catégorie $\mathrm{C}$ ne nécessitant aucun diplôme, 40 \% des recrutés détiennent un diplôme de l'enseignement supérieur).

\section{$\pi$ Ressources documentaires}

\section{Le Conseil d'État}

Le Conseil d'État vient de mettre en ligne la nouvelle édition numérique du Petit Combarnous. II s'agit d'un guide recensant un ensemble de décisions importantes rendue par la haute juridiction tout au long de l'année 2020. La nouveauté de cet ouvrage tient à sa périodicité, désormais annuelle, qui permet un accès plus facile et plus rapide à la jurisprudence du Palais-Royal.

\section{Le rapport annuel de l'Inspection générale des affaires sociales (IGAS)}

Le rapport d'activité 2020 de l'IGAS vient d'être publié. On relève notamment qu'au cours de cette année, I'IGAS a mené 113 missions, dont 36 ont été consacrées, dans des circonstances exceptionnelles, à des appuis à la gestion de la crise sanitaire liée à l'épidémie Covid-19.

On compte 77 missions correspondant quant à elles " aux métiers habituels de l'inspection générale (contrôles, évaluations, audits, appuis classiques) ». Ces missions ont permis d'accompagner des réformes dans les secteurs de la santé (45\% des missions), du travail, de l'emploi et de la formation professionnelle (21\%), de la 
cohésion sociale (18\%) et de la protection sociale (16\%).

\section{EUROPE ET INTERNATIONAL}

\section{$\pi$ Euro}

La poursuite du soutien de la BCE à l'économie

Le 9 septembre 2021, le conseil des gouverneurs de la Banque centrale a décidé à l'unanimité de repousser à décembre une éventuelle révision de sa politique de soutien aux économies. Elle a simplement été légèrement freinée. Les achats de dette, actuellement de 100 Mds€ par mois, vont continuer mais à un « rythme modérément plus faible »: 90 milliards par mois. Les taux d'intérêts restent inchangés.

\section{$\pi$ Politique européenne}

\section{Le blocage du plan de relance hongrois}

Le 6 septembre 2021, les ministres de l'économie et des finances ont approuvé les décisions d'exécution des plans pour la reprise et la résilience de la Tchéquie (7 Mds€) et de l'Irlande (989 millions d'euros). Tout au long du mois de septembre, plusieurs autres plans nationaux ont été approuvés par la Commission qui a, par ailleurs, versé plusieurs fonds de préfinancement. Mais le plan de relance hongrois, soumis à Bruxelles le 12 mai et qui vise à utiliser les 7,2 Mds€ de subventions européennes auxquels Budapest peut prétendre, aurait dû faire l'objet d'une décision de la Commission le 12 juillet, après deux mois d'examen. La période avait été prolongée jusqu'à fin septembre. Le 17 septembre, le premier ministre hongrois a dénoncé un blocage de la Commission en raison des tensions autour du respect de l'État de droit. De la même façon, le plan polonais n'a pas encore été approuvé et, le 4 septembre, le premier ministre Mateusz Morawiecki avait déclaré que, si la Pologne " avait droit » à ces fonds, elle se débrouillait très bien sans et «ne sollicitera pas ces fonds en mendiant ».

Les aides d'État : d'importantes aides illégales

Tout en poursuivant sa politique de large acceptation d'aides nationales visant à amortir les effets de la crise sanitaire, la Commission européenne a déclaré contraire au droit de l'Union plusieurs aides d'État. Ainsi, le 10 septembre 2021, la Commission a constaté que l'apport en capital de
314,6 M€ de PostNord en faveur de PostDanmark ne constituait pas une aide d'État mais que, par ailleurs, le Danemark et la Suède devaient récupérer auprès de PostNord une aide incompatible avec le marché intérieur d'un montant de $66 \mathrm{M€}$. Le même jour elle a aussi considéré que les prêts italiens d'un montant de $900 \mathrm{M} €$ à la compagnie Alitalia (qui a fait faillite en juin 2021) constituent des aides illégales mais que la nouvelle compagnie ITA (Italia Trasporto Aereo), qui reprend certaines de ses activités le 15 octobre 2021, «n'est pas le successeur économique d'Alitalia » (donc elle n'a pas à rembourser les aides illégales à celle-ci) et que les injections de capital de l'État italien dans ITA sont conformes au marché.

\section{INTERNATIONAL}

\section{$\lambda$ Relations multilatérales}

La directrice du FMI et le rapport « Doing business " de la Banque mondiale sur la sellette

Le 17 septembre 2021, la directrice du FMI, Kristalina Georgieva, a été mise en cause, par une enquête interne de la Banque mondiale, pour avoir fait pression en faveur de la Chine et de l'Arabie saoudite, quand elle était responsable du rapport annuel "Doing business » de la Banque mondiale, pour laquelle elle travaillait en 2018. Le changement de méthodes, qu'il avait considéré comme " arbitraire», avait alors poussé à la démission en janvier 2018 le chef économiste de la Banque mondiale, Paul Romer, nobélisé quelques mois plus tard... Le jeudi 16 septembre 2021, la Banque mondiale avait abandonné son rapport « Doing business ».

\section{Monnaies}

\section{Au Salvador, adoption du Bitcoin comme monnaie officielle}

Le mardi 7 septembre 2021, le Bitcoin est devenue une des monnaies officielles du Salvador. Cette innovation n'a pas été sans créer de difficultés. L'application « Chivo » (« super») mise en place pour ces paiements a été suspendue plusieurs fois et la prime de risque sur les emprunts obligataires du pays a augmenté, témoignant des craintes des marchés financiers. Le cours du Bitcoin lui-même ne s'est pas apprécié mais a perdu $10 \%$ de sa valeur. 
La Chine juge illégales toutes les transactions en cryptomonnaies

Le 24 septembre 2021, La Banque centrale de Pékin a déclaré illégales toutes les transactions financières impliquant des monnaies virtuelles. Elle souhaite désormais ainsi lutter contre le "blanchiment d'argent, [les] collectes de fonds illégales, la fraude, et d'autres activités illégales et criminelles ». Cette déclaration a fortement perturbé le cours, très fluctuant de toute façon, des cryptomonnaies, notamment celui du bitcoin.

Aurélien BAUDU (Fiscalité et procédure fiscale),

Fabrice BIN (Europe, international),

Florent GAULLIER-CAMUS (budget de l'État et des opérateurs),

Matthieu HOUSER (finances locales), Christophe SINNASSAMY (management public),

Yves TERRASSE (finances sociales), Robin DEGRON (coordination). $\lambda$ Retrouvez sur www.rgfp.fr :

- l'intégralité des REPÈRES d'actualité des finances et de la gestion publiques depuis 2015 - le 15 du mois, les REPÈRES d'actualité du mois précédent. 